\title{
LLTI Highlights
}

\section{LLTI Highlights}

\section{Ute S. Lahaie \\ Gardner-Webb University}

Welcome to "LLTI-Highlights." This column features a selection of important electronic discussions from the LLTI-Language Learning and Technology International-list server. The discussions of this column were posted during the first half of the year 2005 and have been summarized and paraphrased by me. Otmar Foelsche, the moderator of the electronic discussion list, has assigned a reference number to each topic that appeared in the discussion list. This number can be used to search the LLTI archives. Instructions on searching the archives appear at the end of this column.

A January discussion entitled Copyright: Can Instructors Record Their Audio to Accompany Textbooks/Workbooks? (\#7751) was initiated in December by Fuqiang Zhuo with the following: "I have a few simple questions to ask: 1. Can foreign language instructors record their voice to accompany textbooks or workbooks which are not accompanied by audio and ask the LLC to stream the audio files from its password protected web site? 2. If the textbooks are accompanied by audio files, can we record own version and host on our password protected website. Our professors found that other instructors at other universities have done so. The audio files are not password protected even though there is audio to accompany the textbook." To this, Leslie Pahl replied: Why would instructors want to spend their valuable time rerecording something that already exists to the state of the art? [...] Perhaps you have heard of lawsuits against colleges, universities involving unauthorized distribution over the internet of their materials? If not, my advice would be to not disregard the need for copyright permission." David Flores joined in on the copyright issue: "If you are reproducing copyrighted material (even re-recording the material) you are going to have to get permission to put it on your server from the copyright holder. If you are producing your own materials that are not borrowing from other sources then you should be fine. You own the copyright to these new works." Deanne Cobb added: "...if the students would still need the textbook or workbook for the audio to be of any use, copyright permission should not be too difficult to obtain as the production of the audio would not constitute a loss of profit or sales for the publisher." Francoise Sorgen-Goldschmidt: "Let me address your first question: "Why would instructors want to spend their valuable time rerecording something that already exists to the state of the art?' The reason is that supposedly "state of the art" commercial recordings are often far from satisfactory in quality of reading, 
length of pauses, or for other reasons. Re-recording is something I have done (or found funds for others to do) on several occasions. Back in the time of cassettes, it often seemed that publishers were trying try to fit as much recording as possible into as little time as possible, which would result in unnaturally (ridiculously) fast recording followed by pauses that were frustratingly (to the students) too short. And yes, since the new recordings would go into our collection, I asked permission from the publisher, and they often restricted usage to "Lab only." The same caution (password protection at least) must be exercised with the newer methods available for recording." Judy Shoaf added: "Actually, I know of one case where a professor got permission to record the audio because the official audio was not available at the time. When the latter did become available, instructors liked using both recordings because the professors tended to be slower and easier to understand for beginners (though he is a native speaker). This was done with the copyright owners' permission and in fact they were willing to have us put both sets of recordings online[...]." Similar suggestions came from Zachary Chandler: "...getting permission is the safest thing to do. But what's to stop your faculty from being a little more creative, writing their own scripts, recording original material that covers the same or very similar grammatical or topical areas as the textbook? Have you ever met a faculty member who thought a textbook was perfect as is? This could be an opportunity to complement the text. More work, sure, but you would own it ..."

During the month of February, a lively discussion centered around the topic of Pronunciation evaluation software for German (\#7777). Jing Wang started this thread in January with the following: "A student is interested in using software to evaluate pronunciation in German. She tried to use "101 Languages of the World", yet she was not satisfied with the results. Could you give me some advice and suggestions for available software?" Jack Burston had the following recommendation: "I would suggest having a look at Tell Me More German. It makes quite effective use of voice recognition technology. It is not perfect, but it is one of the best implementations around." Deborah Cordier: I'll second the TELL ME MORE series. I used the French levels this summer at the University of Perpignan in France, and it is very good. Also, Barbara Lafford had an excellent review of TELL ME MORE-Spanish in the September 2004 issue of Language Learning and Technology [...]. The product has been available for some time from Auralog (auralog.org) and is available in several languages, including Arabic." Waltje pointed out his software review of TMM German for the CALICO Review, which can be accessed online at the CALICO website at the following URL <http://calico.org/CALICO_Review/review/tmm-germ00.htm>. Monica Lee had another recommendation: "... 
www.wordchamp.com seems to be a great website that can help you to learn/evaluate pronunciation in German." Ambra Neri cautioned: "If you're only interested in pronunciation evaluation, the Talk to me version is enough and cheaper. It contains the same speaking/pronunciation exercises as the Tell me More series. Tell Me More is the complete program with vocabulary and grammar too. Bear in mind that, while this program is probably the best one available for German, it is not clear what pronunciation aspect the algorithm in it evaluates and how it does it. As a consequence, it is not clear what the score (represented as seven little bricks) is about (speech rate, disfluencies, acoustic quality of single sounds...?). Besides, I suggest you switch off the option that displays the waveforms, since they are only confusing and do not provide any meaningful feedback on the quality of your pronunciation. The pitch display and the red letters are, on the contrary, undoubtedly useful." Barker was also less enthusiastic than the previous responders: "I just tried out a couple of tests here, but wasn't that impressed. There isn't much flexibility in the program. E.g., if you list only the masculine or feminine variant for "cook," the program simply states "Wrong!" in giant letters. Not much of a confidence builder for beginning students! Based on that, I'm not sure that I'd want to pay for their premium service with audio and pronunciation exercises..."

A lively March discussion ensued around the question: Language Labs Living On Borrowed Time? (\#7821). David Flores initiated this discussion with the following: "Our principal Spanish textbook is going to its 6th edition this Summer, and following a trend I've noticed with many FL textbooks lately: the workbook and much of the audio/video content that was traditionally housed in Language Learning Centers is being placed on the web. If this trend continues, much of our 'raison d'être' simply vanishes into cyberspace. Sure we still schedule and show foreign language films in our in-house theatre. Sure we still provide video production and editing facilities for classes that might have a short film project as an assignment. Sure we are open for instructors to run headset-based, interactive class sessions. But most of the use our lab sees... let's face it... is due to students completing their lab manual assignments and listening to the audio-tracks that are stored locally on our server. How many of you are worried about your labs disappearing in five years? And have you any thoughts on how to keep the lab relevant even if textbook manufacturers move most of their audio/video content online?" In response to these concerns, Joseph Kautz wrote: "I am glad someone brought this up. I used to lose sleep over this in the late 90's. [...] Lately, though, I see the issue as an opportunity to be creative and innovative in how to use our Language Lab. Here are some thoughts. Is anyone else rejoicing that publishers are finally getting their acts together around delivering their own digital resources? This is a 
good thing, because it gets rid of a lot of busy work and, if implemented properly. [...] Our lab has a cushion of sorts because our focus is on supporting large scale online assessment. We do online assessment from placement all the way through exiting the language requirement. We are gearing up for our fourth annual online SOPI this spring, which covers eight languages and $600+$ students. This amount of ongoing formative and "informative" assessment requires a facility with a lot of machines that support audio and, soon, video recording of student responses. Sakai (sakaiproject.org) is in beta testing now and will, in my opinion, change the rules significantly, by offering a freely available, enterprise level, open source LMS that is standards compliant. The architecture is modular, allowing different schools to custom make new tools to suit their needs and then share them with other schools. I have seen the mockups and done some testing myself and I can already see a hundred ways the Sakai suite of tools will support foreign language instruction. Much of the activities done in the Sakai application can be done in the language lab. Moreover, the ease with which materials can be made in Sakai will have instructors knocking on my door for content production support..." Anton Brinckwirth added: "Traditional and digital language labs are extremely powerful and effective instructional tools when used competently, inventively, and creatively." Bob Peckham cautioned: "How reliable is the web? One of the reasons I have steered clear of textbooks heavily into web applications is because the web is not always up. [...] If you have a lab, then there are the hubs and there the host server ..." Judy Shoaf added: “... Since at least 1987, when personal cassette players were common, UF has copied (and recycled) lab tapes for students to take home. Now we digitize the material and put it up on a server for students to access at home. So in fact our lab is not very much a place for doing the lab manual audio practice-at most, it's a back-up system. It is however a classroom and examination site, a resource for reserve materials that can't be copied, an information center, and a help desk for the students who can't get the online audio to work. It is also to some extent a technical resource for instructors developing their own materials. We're actually pretty busy...." Read Gilgen: “... Some of us have beaten the publishers to the punch by making our materials online. We've been reinventing ourselves accordingly. Trust me - there is still a lot to do!" Mariana Stone joined in with the following: "The thoughts you just expressed crossed my mind, so I have looked for alternatives about what students can do in our lab and this are the current things that we have going for them:1. Each FL faculty assigns pair work recording as a homework for which the lab tech is required. Instead of just asking written homework from their students and lab manuals in which they only listen now faculty are assigning oral production homework. We developed a feed- 
back system to help faculty respond to the student's recordings. 2 . We enhanced our video library [...]. Videos have become a major source of entertainment for them and therefore learning. The students are expected to watch at least four movies a semester and prepare mini reports on them. Beginning level students can write the mini report in English but they received the whole input of the movie in the target language. 3. Alternative software. We have been purchasing slowly software that helps the students with grammar or pronunciation at different levels of their language learning path. 4 . Songs: we have put our upper level / Spanish education majors to work developing activities based on particular grammar structures covered at the lower levels [...] We encourage the students to complete their lab assignments online from home and have them come to the lab for more resources, which amounts [...] to more contact hours..." Scott Williams also did not consider the publishers' digitized material to be a threat: "...Students prefer to use our lab even for those online assignments for various reasons. These include the fact that we are the only lab that has headphones (and decent ones at that - with mikes if they need them) on all the machines, we guarantee that all the plug-ins, software etc. are installed and work (our campus IT refuses to install RealPlayer on the Windows machines on campus), etc. I am also looking into getting the license to stream some of the material ourselves, but making it available to just our own students. Publisher websites/streaming servers are often overtaxed, cranky, and the tech support unresponsive. They would be smart to let individual campuses set up their own, dedicated parallel streaming servers. The simple fact is, even if you are not developing your own stuff on site, you can tailor your lab to the specific needs of language students in a way many campus-wide IT departments don't care to mess with themselves. Furthermore, you can help your faculty adapt general instructional technology ideas to the specific needs of language pedagogy. What you may lose in physical traffic, you may gain or even double in virtual services." Alexander Waid added: "Actually, in our case, there is no lab manual work done in the language lab: since we're on a closed network, I got permission to put those materials on the campus intranet for my students, they do that at home. The lab is more of a resource center. I and other professors can create or digitize materials, while the students use it with me for class work [...]. They work on writing assignments there. There is a small collection of reading materials and audio/visual materials that are there for perusing, or, sometimes it is assigned as homework. There are programs such as Rosetta Stone for those who want to do some learning/refreshing on their own [...].Students can also do recordings in there. For instance, an English instructor had his students record original poetry in there and then just put all the "submissions" on a CD and gave it out to 
his class so they'd have a copy. If you've got internet connectivity, cultural research can be accomplished in there as part of a day's lesson plan ...." Francoise Sorgen-Goldschmidt: "When I read this message, I somehow recalled reading an excellent analysis on the respective merits of virtual vs. real language labs [...] right in the IALLT Journal (Vol. 36, Number 1 2004) by Peter Yang." Jeff Samuels wrapped up the discussion with in the following posting: "WOW! What a flurry of replies! [...] I do wish to address the point you made that the textbook publishers are putting more and more online and hosting materials themselves. This is true, but we're not out of the woods yet! As Digital Exchange Coordinator for IALLT, I am particularly anxious to see this trend continue, but I have to say that we are far from the point where we can rely on the publishers to provide this service in a way that is dependable and consistent for our members. To wit, we still have to take into account: differences in file formats; permissions and restrictions, authentication of users; usability and meta-data (What good is it to students when there is no list of exercises by title and page number, and instead they have to match up a file entitled "track 34 " with their assignment?), and of course, bandwidth and speed concerns. We can address every one of these when publishers allow us to host materials on our own servers and networks, and to make index pages that guide the students to the materials they need. So, my sense is that the language lab / learning center / resource center as we know it is an evolving animal; yet there are certain services that many of us perform that we will continue to engage in until connectivity, security, ease of access, support, dedicated space needs, and other factors undergo a significant transformation. Let me close by seconding the many previous responders who have pointed out the increasing depth and complexity of our services to our students and colleagues."

An April discussion on LangLab and NetSupportSchool Reviews (\#7877) was launched by Daniel Wescovich with the following query: "Is anyone currently using the LangLab digital language lab software, and if so, can you give me any feedback on this particular program and its performance in the language lab classroom? Also, is anyone using NetSupportSchool in their language labs, and if so, do you have any feedback on its performance in the classroom? [...] Is it a reasonable software solution for a digital language lab classroom? Doug Worsham immediately responded with the following: "We use NetSupport School (NSS) in our labs and have been happy with it. We use it primarily for transferring files, launching programs on the client computers, and viewing, displaying, and locking client screens. While it does have some audio capability, it doesn't make grouping and pairing students for speaking activities particularly easy, and we've found that there is quite a bit of audio delay in the current version, so we use other software applications for audio work. 
[...]We've found that NSS used in concert with free audio applications is an economical and flexible solution for language labs. However, if you're looking for an entirely integrated package - with all the bells and whistles for recording and pair/group discussion in a single program, the current version of NSS may not meet your needs." Jack Burston pointed out a resource: "I would suggest getting a copy of the Digital Language Lab Solutions volume, soon to be available from the IALLT website. It contains a comprehensive evaluation of LangLab and NetSupportSchool as well as nine other programs that may be of interest to you." Read Gilgen cautioned: "NetSupport School (NSS) isn't a perfect solution, but we use it and find it works well for the things we need it for (mostly lab control software). [...] As for the audio portion... yes it works, and fairly well, for All Call and one-to-one (teacher/student) communications. Teachers can also just listen. Because the audio is over IP, there is a slight delay (latency...." To this, Chen Xiaobin responded: "I think the audio delay must have something to do with your net rate. The software designing theory and algorithm play important roles on the final effect of its performance. But this is based on the good performance of the facilities that run the software. So the device, equipment and net connection rate also need to be considered carefully. And I have a question. Do you use only computer lab as language lab? I am in China, and in my university the language labs are installed with computers and also special units. That is to say, a computer controls the displaying and listening unit for each student. The unit is not a computer, but it seems easy for clear sound to transfer without any delay. I don't know whether this kind of setting is better for a language lab." Read Gilgen: "We use only the computers. We wanted to avoid being tied to proprietary hardware."

Mark Lauersdorf started another discussion on technical issues during the month of May with his inquiry concerning the Canopus analog-digital converters (\#7900): "Does anyone have any direct experience with Canopus video conversion products? We are considering purchasing the $\mathrm{ADVCl} 10$ as the only video conversion device that we would own in our Language Learning Center. We are looking for something that will be Mac-PC cross-platform compatible and a desktop unit (not a card) so that it is portable. The Canopus ADVC1 10 (<http://www.canopus.us/US/products/ADVC110/pm_advcl10.asp $>$ ) has gotten good reviews $[\ldots]$, but we are also wondering, if the ADVC300 (<http://www.canopus.us/US/products/ADVC300/pm_advc300.asp >) is worth the extra money or whether the added features go beyond what we would need to do basic good-quality analog-digital conversion..." A first recommendation came from Tom Browne: "I have been using the 110 for the last year. It seems to do the conversions both ways well. Unless you are doing professional, broadcast work it should do the trick." Claire Sandler reported: "I have been using the Canopus ADVC-100 with an iMac since September, and so far it has worked 
flawlessly! Although it isn't technically supposed to, I have even hooked it up to a DVD player to import dvds (in-house, of course,) for re-editing with iMovie. One drawback is that the manual offers no tips on how to use the converter." Derek Roff added: "We have been using the Canopus ADVC300 for about a year, and have been happy with it[...]The converted digital footage shows no significant degradation from the analog original. In fact, I would say that the two are indistinguishable, in most cases. Close analysis of scenes with a lot of random movement will sometimes show some digital artifacts. This is most likely to be present when the original image is degraded, with significant color fringing. When the original analog video has high quality, the digital conversion is excellent. I have no direct experience with the ADVC110, so I can't compare it to the ADVC300."John F Niendorf and Victor Aulestia also reported positive experiences with the converter. Zachary Chandler joined in: "I have a handful of Canopus 100's in my lab, and I can attest to the positive reviews you have read. I have only used them with iMovie and FCP; I can't comment on Windows compatibility...."

A June discussion on Web-based audio recording applets/tools (\#7924) was started in May by Samantha Earp with the following: "I am writing to see if any of you know of Flash/Java applets or other tools that allow one to record voice from within a Web page or application. I am already aware of Wimba and Edufolio, which uses a Flash applet \& Flash Communication Server, but I would like to know if there are any other possibilities out there, particularly ones that don't require the purchase of an entire wrapper or course management-type system to use..." Dennie Hoopingarner pointed out another product: "We at Michigan State University's Language Learning Center developed a Flash-based program called Audio Portfolios that does exactly what Samantha describes in her post. It is a stand-alone tool that allows teachers to create accounts for their students to record audio (and video) clips, store them centrally on our server, and make them available to teachers. The program is available on a subscription basis. For $\$ 50$, instructors can make any number of class lists with any number of student accounts. Students do not have to pay for access, as they log in under the account that their instructor creates for them. You can try the program at the website listed below. Under the FAQ section there is documentation in PDF format, and the Licensing section details how to purchase accounts <http://distancelearning.llc.msu.edu/audioportfolio> ..." Dick Feldman: "At Cornell we have developed a program called Web Audio Lab. It was written in Macromedia Director by Slava Paperno, from our Russian Language program. The client, CD-based program records locally and then batch submits files to a server. He also wrote a module that allows recording from a web page, which we have integrated into the web site of this program to allow recording 
of teacher comments back to students. It is also being used in other web applications to allow student recording. You can see some more description of this program at our Showcase page: <http://lrc.cornell.edu/showcase $>$." Jack Burston recommended the latest IALLT publication: "I would suggest getting a copy of the recently published IALLT Digital Language Lab Solutions volume. It provides a comprehensive evaluation of eleven programs including a number that may have the features you're looking for." Richard Laden had the following suggestion: "LangLab [...] will, of course, also allow students to record over the Internet and compare what they record to model utterances. Among its advantages are the integration of student responses/recordings with the model to facilitate comparison (no need to switch back and forth), and the flexibility of lesson items, which can be short or long, can have one model and response or many, etc. LangLab is a cross-platform solution that also allows teachers to retrieve and insert comments in students' recordings, track students' progress at a glance, and has modules for real-time monitoring of a class (including remotely, for a virtual lab) and lesson creation. We put another fully-functional demo version with documentation (release 1.8) on our site <www.elanglab.com> about one month ago."

The discussions of the Language Learning and Technology International (LLTI) list server have been archived and posted on the web. These LLTI archives can be accessed from the IALLT Home page, which is located at <http://www.iall.net>. A link to the LLTI list server appears at the top level of the IALLT Home page. The LLTI list server page gives instructions on how to subscribe or unsubscribe to the list server. In addition, there is a link to the LLTI archives. These archives can also be directly accessed at <http://listserv.dartmouth.edu/archives/llti.html>.

To search the archives, type the subject in the first search field. The search engine will match the subject with the subject headings of the archived messages. You may also type a key word or words in the second search field. This search will look for the key words in the body text of all messages. It the reference number that has been assigned to a discussion topic is entered in this field, the search will bring up a complete list of all discussions dealing with the topic. You may also want to restrict search by limiting it to a specific author or by entering beginning and ending dates. Such a restriction is particularly useful for searches on frequently occurring topics. Any questions or comments related to the LLTI list server may be addressed to Otmar Foelsche, the moderator of the LLTI discussion list. Otmar can be contacted at otmar.foelsche@dartmouth.edu. 


\section{LLTI Highlights}

Ute S. Lahaie is Associate Professor of German at Gardner-Webb University. She serves on the IALLT Board as

Secretary/Parliamentarian. 